\title{
Research on the Prediction of Stock Market Based on BP Neural Network
}

\author{
Yaze Yuan $^{1}$,* Mengmeng Su \\ ${ }^{1}$ Northwestern Polytechnical University, Xi'an, 710000, China \\ ${ }^{2}$ Shanghai ZhiziNetwork Technology Co., Ltd., Shanghai, 200000, China \\ *Corresponding Author: Yaze Yuan
}

Keywords: Prediction technology, Stock market, BP neural network.

\begin{abstract}
The stock market is a highly complex nonlinear dynamic system, and its fluctuation rule is affected by many factors. The index prediction of the stock market is a concern of economic managers. This paper first analyzes the concept and characteristics of BP neural network, and then proposes a prediction model of Shanghai composite index based on BP neural network. The result shows that this scheme has higher accuracy, which can provide some references for the investors to guard against the financial risks.
\end{abstract}

\section{Introduction}

The securities market has the dual characteristics of income and risk, and the return of stock investment is proportional to the risk. Generally, the higher the investment income, the greater the risk. As more and more people enter the stock market for investment activities, there is an urgent need for an effective analysis method that can maximize revenue and reduce risk. Therefore, the research of stock market prediction method is of great theoretical significance and practical value. Neural network is a hot research field in recent years. It has a great attraction to the research of artificial intelligence with new and different ways of expressing and dealing with information. The securities market as an important part of market economy, to a certain extent reflects the overall economic trend of a country, so the stock market forecast is an important field of economic development of countries in recent years. With the development of global economic integration, the world increasingly pays more attention to the securities market to play a role, and the inherent laws of the stock system always in a special attitude influencing and changing people's life. In the process of establishing the securities system, price forecasting is always an important factor affecting the development of the securities market. But the securities system itself is a highly complex and difficult to determine linear system. Traditionally, diagrams were the basic tool for technical analysis. Chart information has obvious advantages, but the analysis and index selection depend on subjective judgment. This is the main dilemma faced by traditional technical analysis. Because of the complexity of securities trading patterns and related information, the reliability of such a highly empirical theory or approach is questionable to a large extent. Therefore, the price forecasting in the stock market is often affected by uncertain factors. Therefore, it is necessary to explore the important role of the neural network method in the prediction of the stock market.

\section{Concept and Features of BP Neural Network}

Concept. A neural network, also called an artificial neural network, is a parallel, distributed processor network consisting of many connected simple neurons. Like the human brain, the neural network, to some extent, has the function of storing, computing and using, or the neural network is abstract human brain. The neural network model is based on the biological reaction between biologists developed a set of intelligent information processing model, the main function is to use its own algorithm to receive information from the outside world, the program system through each 
neuron transfer and operation to obtain the required calculation target. A neural network is a parallel distributed system consisting of many simple processing units and connected by variable weights. Neuron is the basic processing unit of artificial neural network. It is a nonlinear device with multi input and single output. According to the characteristics and functions of neurons, neurons can be abstracted into a simple mathematical model. BP network is a multilayer feed-forward neural network, usually three or more than three layers of neural networks, including input layer, intermediate layer and output layer. Full connection is achieved between the upper and lower layers, and no connection between the layers. The basic component of the artificial neural network is the neuron, which is called the processing unit. The biological structure of a single biological neuron is simple, its processing time is not very fast, and the way of processing information is simple. But the biological brain contains many neurons, and each neuron is connected to tens of thousands of other neurons, making the overall system very fast. The transfer function of a neuron is a $\mathrm{S}$ type function with a continuous quantity between 0 and 1 . It can achieve any nonlinear mapping from the input to the output.

Features. BP neural networks are characterized by large-scale parallel processing, distributed storage, resilient topology, highly redundant and nonlinear computing. So, it has very high computation speed, good association ability, strong adaptability, fault tolerance and self-organization ability. Its neural network has good properties and characteristics. The information processing method of neural network is to simulate the thinking of human brain. The human brain contains many neurons, and the connection between cells is very strong. Therefore, the human brain can deal with nonlinear problems, which determines the nonlinear characteristics of the neural network. For different environments, the neural network is not a fixed system, the human brain's thinking is moving, and when the outside has different input of information, the neural network will constantly correct itself. The human nervous system is non-local, for example, a single neuron does not determine the final behavior of the human body, and the behavior of the human body is a composite result of the overall functioning of the neurons. The global performance of neural networks can be represented as distributed storage. Non-convexity means that the neural network does not have the only equilibrium point. In a specific range, it may converge to the local minimum point, but not the global minimum. This requires a global optimization of the neural network. These characteristics and capabilities constitute the technical basis for artificial neural networks to simulate intelligent activities, and have gained important applications in a wide range of fields. BP neural network is the most widely used neural network. It has the ability of nonlinear mapping, generalization and fault tolerance. It is the preferred neural network for stock prediction analysis. We introduce BP neural network technology into stock investment risk analysis and evaluation to predict the stock price trend and promote the healthy development of China's economy.

\section{Prediction Model of Shanghai Composite Index Based on BP Neural Network}

Data Acquisition. Stock is a very unstable market, and the individual stocks are prone to speculation. If the selected samples are not typical, the generalization ability of neural networks will be reduced. Therefore, the selection of samples is critical. Choosing Shanghai stock index as a learning sample mainly has one factor: first, the Shanghai stock index is the weighted average value of all stocks, and relatively objectively reflects the stock market law. First, the individual stock is very vulnerable to the main capital speculation, cannot really reflect the volatility of the stock, while the Shanghai composite index is relatively stable. Second, the Shanghai Composite Index has the most obvious impact on investors, so it is important to predict the Shanghai Composite index. Four, the Shanghai composite index reflects the dynamics of the domestic financial market and objectively reflects the situation of the domestic macroeconomic. The Shanghai composite index is the abbreviation of the Shanghai stock exchange stock price composite index, which is a stock index compiled by the Shanghai stock exchange. The stock index is a sample of all stock listed on the Shanghai stock exchange, where new shares are included in the stock index after the listing day. In China's stock market, the Shanghai stock index has long been the most influential and investors' most concerned index. It represents the macroeconomic situation and the trend of market operation. The 
Shanghai index is a reporting period the number of shares issued for stock index weighted comprehensive weights, which contains as many as stocks only, can fully represent the entire market price movements, it is difficult to be controlled, which can be reflected in the stock market rules operation. This paper selects stock index of Shanghai stock market to predict. The original data is the closing price, volume, and a series of technical index time series of the Shanghai Composite Index from March 1, 2016 to July 31, 2016.

Index Selection. The essence of the technical index analysis method is to calculate the technical index by mathematical method, which reflects the law or connotation of a certain aspect of the stock. It is difficult to see through the original data. Based on technical analysis of the market behavior is assumed to contain all the information, the stock price influence factors whether the macro economy, policy, psychology or any other aspects of the various, are reflected in the price change, which can be of various factors affecting the operation of stock price into the stock price change on the study of history. Stock price, volume and technical analysis indicators are the best interpretations of market behavior, among which price and volume are the most basic and core factors. The index value of moving average is the average price of the closing price of stock trading day, and the moving average mainly plays the role of trend guidance and support, and pressure. Reflects the average value of stock prices over a period. As the stock price changes, the average price fluctuates. Because the moving average is generated by the continuous movement of a set of samples, its moving direction and speed represent the overall situation of the set. Volume refers to the amount of transactions a transaction is made within a time unit. Under normal circumstances, large volume and rising prices of stocks, the trend for the better. When trading volume remains low, it is generally in the bear market or stock consolidation stage, and market transactions are inactive. Volume is a supply and demand performance. We quantify this crowd, that is, the volume. Turnover is an important basis for judging stock movements, and provides an important basis for the analysis of the main behavior. The psychological line is based on a study of investors' psychological tendency within a certain period the buyer or seller's psychology and reality into numerical form as a parameter of stock trading.

Data Processing. We can design filters to filter outliers under linear conditions, but they cannot be easily filtered under nonlinear conditions. These outliers themselves also contain valuable information. They may indicate a change in a pattern or structure. As the data contains the outliers, the robustness of the system is required. To avoid the difference between the magnitude of the raw data or the numerical value between the different indexes, we need to normalize the raw data. For predictive values, we also hope that their output results are within a fixed range. Therefore, the output of the neural network also needs to be processed with the necessary normalization. Normalization is to fix the range of the original data within a range. In this system, the neural network transfer function is based on the type of activation transfer function. Therefore, it is necessary to normalize the raw data to between 0 and 1 , and the concrete method is to find the maximum value $X_{\max }$ and the minimum value $X_{\min }$ of the original database. $X_{i}$ is the function of $X$. The data after the normalization is:

$$
X_{i}=\frac{X-X_{\min }}{X_{\max }-X_{\min }}
$$

Due to the normalization of the network inputs, the output of the network is not the direct price information. It also needs to deal with the output of the network, making the processed results are consistent with the range of stock price. The process is called anti normalization, whose formula is:

$$
\mathrm{X}=X_{\min }+\left(X_{\max }-X_{\min }\right) X_{1}
$$

Experimental Results. For neural networks, the differentiability of the transfer function is very important. The neural network toolbox provides three different transfer functions, which are suitable for the type function between input and output. We enter the sample data and normalize the data. The experiment results are obtained by the Matlab. 
Table 1. Comparison of actual value of Shanghai composite index and predictive value of Shanghai composite index

\begin{tabular}{|c|c|c|c|}
\hline Number & $\begin{array}{l}\text { Actual value of Shanghai } \\
\text { composite index }\end{array}$ & $\begin{array}{l}\text { Predictive value of Shanghai } \\
\text { composite index }\end{array}$ & Error percentage \\
\hline 1 & 3194.2 & 3180.5 & $-0.4 \%$ \\
\hline 2 & 2916.3 & 2903.7 & $-0.4 \%$ \\
\hline 3 & 2980.3 & 2971.5 & $-0.3 \%$ \\
\hline 4 & 3006.5 & 2990.3 & $-0.5 \%$ \\
\hline 5 & 2955.7 & 3013.5 & $2.0 \%$ \\
\hline 6 & 2980.3 & 3000.1 & $0.7 \%$ \\
\hline 7 & 2890.6 & 2900.6 & $0.3 \%$ \\
\hline 8 & 3112.4 & 3179.1 & $2.1 \%$ \\
\hline 9 & 3024.8 & 3100.9 & $2.5 \%$ \\
\hline 10 & 3109.1 & 3100.8 & $-0.3 \%$ \\
\hline
\end{tabular}

BP network has strong robustness, good self-learning and fault-tolerant ability. The convergence rate of BP network is faster than that of momentum BP network. The BP network has a certain accuracy to the Shanghai composite stock index prediction. It can provide reference for investors to guard against the financial risks.

\section{Conclusion}

The predictability of stock price proves that China's stock market has its inherent law, and the operation of Shanghai composite index is not disorder. In this paper, BP neural network model is used to forecast stock price, and good results are obtained. However, the stock market is a constantly changing dynamic system, and the network system must be trained many times according to the new data produced by the stock market to adapt to the complicated situation.

\section{References}

[1] Shi Lei. Predictionanalysisof SSE compositeindexbased onoptimized BP algorithm [J]. Journal ofFuyangTeachers College (Natural Science), 2013, 30(1): 58-62.

[2] Xu Mei, Wang Fang. Forecasting Financial VolatilityBased on BP NeuralNetwork andSymbolic Time Series [J].Journal of Wuhan University of Technology (Information \& Management Engineering), 2015, 37(4): 456-460.

[3] Shi Lei. Predictionanalysisof SSE compositeindexbased onoptimized BP algorithm [J]. Journal ofFuyangTeachers College (Natural Science), 2013, 30(1): 58-62.

[4] Huang Hongyun, Wu Libing, Li Shizheng, LyuShishan, Liu Qi. An improved IPSO-BP neural network in stock market index forecasting - A casestudyof Shanghai Composite Index [J].Journal ofYanbian University (Natural Science), 2016, 42(4): 351-356. 